\title{
Saneamento básico e seus impactos na mortalidade infantil e no desenvolvimento econômico da região Nordeste
}

Basic sanitation and its impacts on infant mortality and economic development in the Northeast region

\author{
V. Andrade Silva ${ }^{*} ;$ F. Esperidião ${ }^{1}$ \\ ${ }^{1}$ Núcleo de Pós-Graduação em Economia (NUPEC), Universidade Federal de Sergipe, 49100-000, São Cristóvão- \\ Sergipe, Brasil
}

*valeria.andrade81@hotmail.com

(Recebido em 29 de maio de 2017; aceito em 28 de setembro de 2017)

\begin{abstract}
O saneamento básico é um fator de relevância para a saúde e para as questões socioeconômicas. Este estudo foi realizado com o objetivo de analisar os impactos do saneamento básico sobre a mortalidade infantil e o desenvolvimento econômico dos estados do Nordeste. Para tanto, foram estudados os serviços de abastecimento de água e esgotamento sanitário, como também a mortalidade infantil e algumas variáveis que refletem as questões socioeconômicas da região Nordeste no período de 1991, 2000 e 2010. Os dados foram coletados do IPEADATA e do PNUD. Os resultados foram obtidos a partir da análise descritiva, estatística, da matriz de correlação e dos gráficos de dispersão gerados pelo software STATA 12. Concluise que investimentos em abastecimento de água e esgotamento sanitário podem reduzir tanto a mortalidade infantil quanto as iniquidades sociais.

Palavras-Chave: água e esgoto inadequados, doenças hídricas.
\end{abstract}

Basic sanitation is a factor of relevance for health and socioeconomic issues. This study was carried out with the objective of analyzing the impacts of basic sanitation on infant mortality and economic development in Northeastern states. For this purpose, water supply and sanitary sewage services, as well as infant mortality and some variables that reflect the socioeconomic issues of the Northeast region, were studied in the period 1991, 2000 and 2010. The data were collected from IPEADATA and PNUD. The results were obtained from the descriptive, statistic analysis, the correlation matrix and the dispersion graphs generated from the software STATA 12. It can be concluded that investments in water supply and sanitary sewage can reduce both infant mortality and social inequities.

Key-words: inadequate water and sewage, water diseases.

\section{INTRODUÇÃO}

O saneamento básico se constitui em medidas de abastecimento de água, esgotamento sanitário, limpeza e manejo de resíduo sólidos e drenagem e manejo de águas pluviais urbanas. Estes serviços promovem a saúde pública por meio da prevenção de doenças infectocontagiosas e se constituem em um direito social para a população, sendo relevantes na promoção da qualidade de vida e do bem-estar socioeconômico [5].

Segundo Leal (2012) [1], quanto maiores os investimentos em saneamento, menores serão os gastos públicos com saúde. Tendo impacto sobre a capacidade produtiva de cada indivíduo e o desenvolvimento da região.

Com relação a mortalidade infantil, o saneamento é um fator de relevância na diminuição dos óbitos [3]. Pois, as doenças diarreicas são as principais causas dessa mortalidade. Isso ocorre porque as crianças perdem nutrientes importantes na idade em que mais necessitam para sobreviver. Sendo que a implementação dos serviços de abastecimento de água e esgotamento sanitário são as formas de prevenção destas doenças [4,1]. Por este motivo, neste trabalho é dada maior atenção aos serviços de abastecimento de água e esgotamento sanitário.

Para Turolla (2014) [9] o déficit em saneamento atinge em maior nível as regiões menos desenvolvidas, atrasando-as ainda mais no seu processo de desenvolvimento. Tanto as concentrações dos maiores índices de serviços de abastecimento de água e esgotamento sanitário 
inadequado quanto os maiores números de mortalidade infantil, estão localizadas nas regiões em que há menor Índice de Desenvolvimento Humano, ou seja, aquelas regiões em que há menor nível de escolaridade, renda e acesso aos serviços de saúde.

Neste contexto, o estudo analisa o impacto dos serviços de abastecimento de água e esgotamento sanitário na mortalidade infantil e no desenvolvimento econômico dos estados da região Nordeste no período de 1991, 2000 e 2010.

\section{MATERIAL E MÉTODOS}

Para alcançar os objetivos propostos, foi realizado um levantamento bibliográfico para a fundamentação teórica e para dar embasamento aos resultados obtidos a partir dos dados secundários das variáveis: Mortalidade Infantil (MI); Percentual de pessoas em domicílios com abastecimento de água e esgotamento sanitário inadequado (AgEsI); PIB per capita - R \$ - 2010 (PIBp); Percentual da população de 18 ou mais anos de idade com fundamental completo (TFC); Índice de Gini (IG); Índice de Desenvolvimento Humano (IDH) e Despesas com saúde e saneamento - R \$ - 2010 (DSS). De acordo com Menezes e Uchoa (2011) [3] e Sousa (2016) [8], as questões socioeconômicas impactam na mortalidade infantil e no desenvolvimento regional.

Com relação aos dados, os mesmos foram coletados para os períodos de 1991, 2000 e 2010, do Atlas do Desenvolvimento Humano no Brasil do Programa das Nações Unidas para o Desenvolvimento (PNUD) e do Instituto de Pesquisa Econômica Aplicada (IPEADATA), para os estados da região Nordeste. Os resultados foram alcançados a partir da análise descritiva e estatística, da matriz de correlação e dos gráficos de dispersão gerados no software STATA 12.

\section{RESULTADOS E DISCUSSÃO}

Para uma análise preliminar, as Figuras 1 e 2 mostram os índices de pessoas em domicílios com abastecimento de água e esgotamento sanitário inadequado e o número de mortalidade infantil (número de crianças com até um ano de idade que não deverão sobreviver a cada 1000 crianças nascidas vivas). As figuras permitem ter uma visão espacial da distribuição desses índices pelos estados do Nordeste, o que facilita a visualização da possível relação que existe entre eles. De acordo com Mendonça e Motta (2007) [2], o saneamento básico possui relação direta com a mortalidade infantil, pois a falta dos seus serviços ou a sua prestação inadequada impacta no aumento do número de mortalidade infantil.

Na Figura 1, percebe-se que houve diminuição dos índices de água e esgoto inadequados ao longo do período, sendo essa diminuição causada possivelmente pelo aumento dos investimentos em saúde e saneamento feitos pelo governo, o aumento da renda per capita e do nível de escolaridade da população.

Observa-se que o maior índice de água e esgoto inadequados foi de aproximadamente $36 \%$ em quase todos os estados do Nordeste em 1991, com exceção apenas dos estados de Sergipe e Pernambuco, os quais tiveram um índice entre $15 \%$ e $25 \%$. No ano de 2000, os índices tiveram uma diminuição variando entre $5 \%$ e $30 \%$, com destaque para o estado do Piaú que teve uma queda de 28,2 pontos percentuais (p.p). Já no ano de 2010, os índices variaram entre $5 \%$ e $20 \%$ aproximadamente. $\mathrm{O}$ estado de Sergipe, por exemplo, teve uma queda de 5,17 p.p no período. Uma queda que parece pequena, mas quando observada na Figura 2, percebe-se que foi representativa, uma vez que no mesmo período, o número de mortalidade infantil sofreu uma queda de 13,45 mortes para cada 1000 crianças nascidas vivas.

Nota-se que a mortalidade infantil sofreu uma queda significativa, passando de uma variação entre 60 e 85 mortes em 1991, para uma variação entre 19 e 30 mortes aproximadamente para cada 1000 crianças nascidas vivas em 2010. De acordo com Pasternak (2016) [5], essa diminuição do número de mortalidade infantil se deu pela diminuição dos serviços inadequados de saneamento, uma vez que o abastecimento de água e o esgotamento são os meios de prevenção das doenças infectocontagiosas, não permitindo a sua transmissão.

Para Ramalho (2014) [7], essa diminuição pode ter ocorrido também pelo aumento do nível de escolaridade da população. Já que quando há maior nível de alfabetização, há uma tendência 
de a população ter maior consciência dos seus direitos, como também da importância do saneamento básico e da higiene pessoal para a saúde tanto das crianças quanto dos adultos e para a qualidade de vida. Apesar de ter ocorrido uma diminuição dos serviços inadequados e do número de mortalidade infantil na região Nordeste, essa ainda continuou a ser uma das regiões que possuíam maiores índices, ficando atrás apenas da região Norte, isso se deu pelas grandes desigualdades sociais e econômicas da região [3].

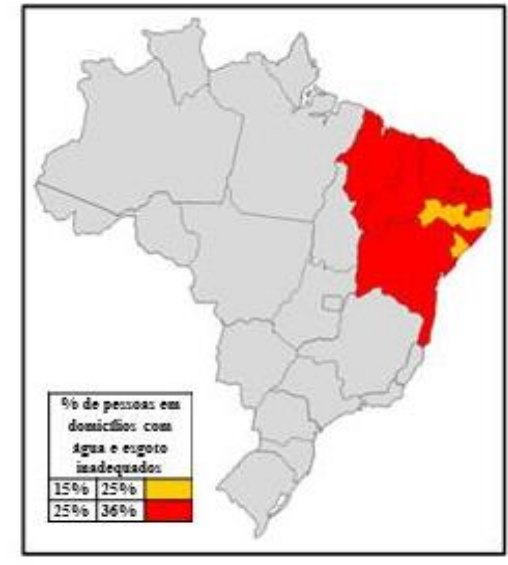

a

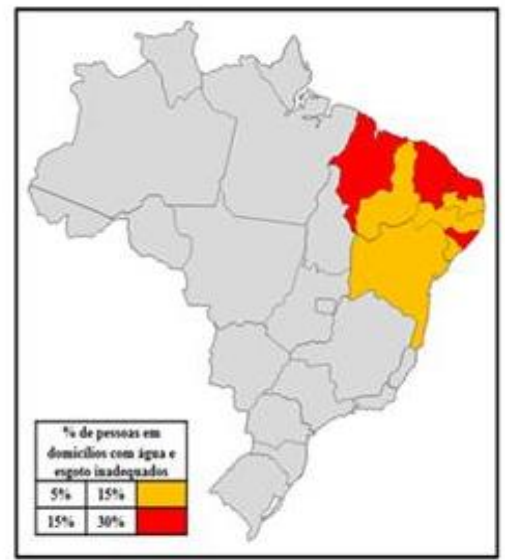

b

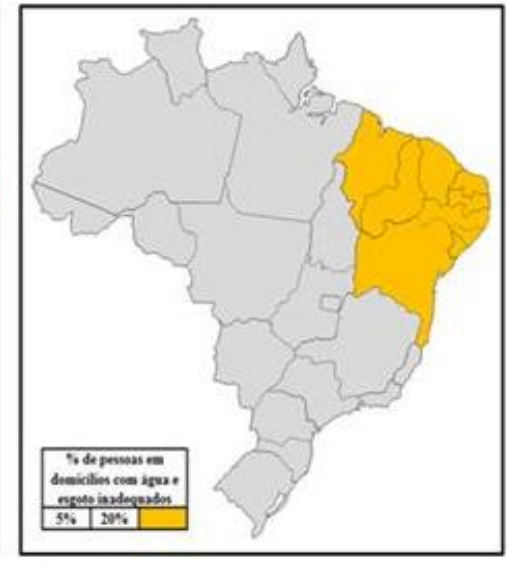

c

Figura 1 - Percentual de pessoas em domicílios com abastecimento de água e esgotamento sanitário inadequados - (a) 1991; (b) 2000; (c) 2010

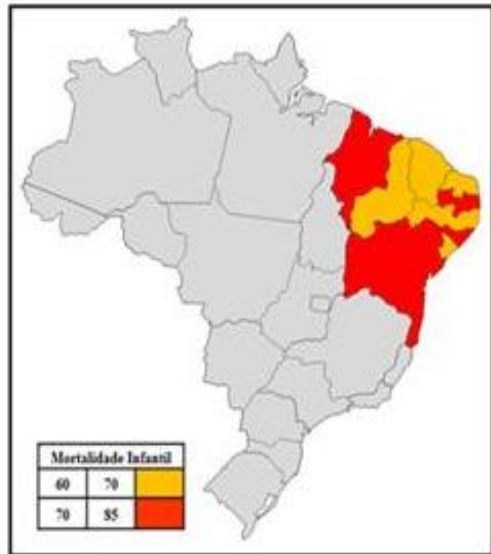

a

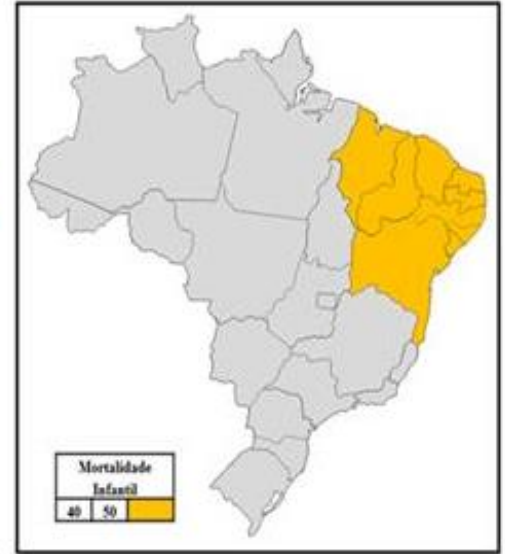

b

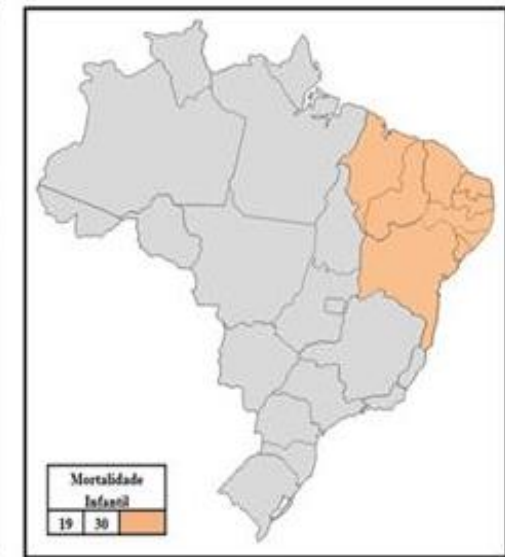

c

Figura 2 - Mortalidade infantil - (a) 1991; (b) 2000; (c) 2010

Destarte, na Tabela 1 são apresentadas as estatísticas descritivas sobre a mortalidade infantil e as variáveis selecionadas para o estudo com o intuito de observar as condições socioeconômicas dos estados. A média do número de mortalidade infantil na região Nordeste foi de 45,47 óbitos infantis, sendo que o menor número apresentado foi no estado do Ceará com 19,29 mortes. No entanto, o maior número $(81,97)$ foi observado no estado do Maranhão, o qual também apresentou maiores desigualdades sociais. Em relação ao percentual de pessoas em domicílios com abastecimento de água e esgotamento sanitário inadequado, a média foi de $18,9 \%$, sendo que o menor índice foi observado no estado do Piauí $(7,31 \%)$. O estado que apresentou maior Índice de Desenvolvimento Humano foi o Rio Grande do Norte com 0,68, o qual também apresentou bons índices de pessoas com 18 anos ou mais com fundamental completo. Contrariamente a isso, o estado de Alagoas apresentou a maior concentração de renda 0,68 , salientando que quanto mais próximo de um for o índice, maior é a desigualdade de renda. Em relação às despesas com saúde e saneamento, percebe-se que houve um aumento significativo nos estados do Nordeste, sendo que o maior investimento realizado no período foi no estado da Bahia com $\mathrm{R} \$ 4.111 .582 .926,06$. 
Tabela 1 - Estatísticas descritivas

\begin{tabular}{cccccc}
\hline Variáveis & Observações & Média & Desvio Padrão & Mínimo & Máximo \\
\hline MI & 27 & 45,477 & 19,9733 & 19,29 & 81,97 \\
AgEsI & 27 & 18,9053 & 8,8505 & 7,31 & 35,89 \\
IG & 27 & 0,6362 & 0,022 & 0,6 & 0,68 \\
PIBp & 27 & 7,3459 & 2,0645 & 4,26 & 11,57 \\
TFC & 27 & 31,634 & 10,5792 & 18,24 & 48,83 \\
IDH & 27 & 0,5214 & 0,114 & 0,357 & 0,684 \\
DSS & 27 & $6,30 \mathrm{e}+08$ & $1,03 \mathrm{e}+09$ & $4,696,97$ & $4,111,582,926,06$ \\
\hline
\end{tabular}

Com relação à Tabela 2, têm-se as correlações das variáveis percentual de pessoas em domicílios com abastecimento de água e esgotamento sanitário inadequado (AgEsI); PIB per capita - R \$ - 2010 (PIBp); Percentual da população de 18 ou mais anos de idade com fundamental completo (TFC); Índice de Gini (IG); Índice de Desenvolvimento Humano (IDH) e Despesas com saúde e saneamento - R\$ - 2010 (DSS) com a Mortalidade Infantil (MI). Todas as variáveis apresentaram correlação com a MI, assim como os sinais esperados pela literatura. Segundo Neri (2007) [4], a relação entre a saúde e o desenvolvimento é estreita, sendo uma condição da outra. Para Menezes e Uchoa (2011) [3], essa relação existe, pois quando a população está saudável ela é mais produtiva. O que impacta de forma positiva no desenvolvimento, já que há menor número de faltas no trabalho e nas escolas. Além disso, o governo diminui os gastos com saúde, já que os gastos com tratamentos de doenças ou mesmo prevenção diminuem. Essa relação pode ser o fator explicativo das diferenças de desenvolvimento dos países, uma vez que o acesso a saúde é limitado ou quase nenhum dentro das desigualdades sociais. Observa-se ainda na Tabela 2, que os valores apresentados são significativos, pois apresentam desde correlação fraca a correlações muito fortes como, por exemplo, a correlação entre a MI e o AgEsI, que apresentou uma correlação de 0,8129 . O que reflete que possivelmente quando houverem maiores investimentos em saneamento, isso poderá ocasionar melhorias na saúde, nos aspectos socioeconômicos e na diminuição da mortalidade infantil.

Tabela 2 - Matriz de Correlação

\begin{tabular}{cccccccc}
\hline & MI & AgEsI & IG & PIBp & TFC & IDH & DSS \\
\hline MI & 1 & 0,8129 & 0,3692 & $-0,7126$ & $-0,9361$ & $-0,9743$ & $-0,6638$ \\
\hline
\end{tabular}

As Figuras a seguir apresentam os gráficos de dispersão, nos quais constatam-se que as relações entre as variáveis são não lineares e que quanto maior for a correlação, mais juntos serão os pontos do gráfico, formando quase uma reta. Assim, na Figura 3, é possível visualizar a relação entre a mortalidade infantil-MI e os serviços de água e esgoto inadequado-AgEsl, a qual é positiva. Demonstrando assim, uma relação direta, pois um aumento em AgEsl pode impactar em um aumento em MI. 


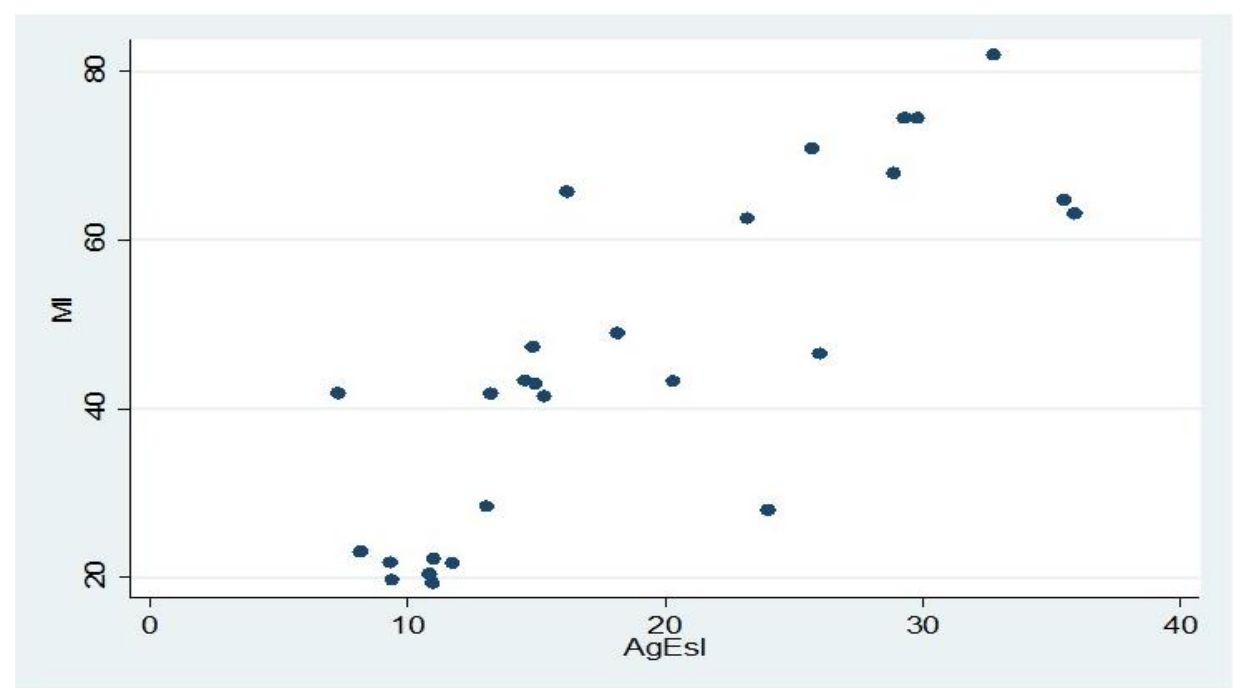

Figura 3 - Gráfico de Dispersão - MI-AgEsI.

Na Figura 4, é apresentada a correlação entre as variáveis MI e PIBp, onde percebe-se uma relação negativa e forte, refletindo que, quando há um aumento no PIBp, pode ocorrer uma queda em MI. Já que quanto maior for o PIBp da região, supõe-se que existam maiores investimentos em saúde, educação, trabalho entre outros, que refletem em diminuição das iniquidades sociais e do número de óbitos infantis.

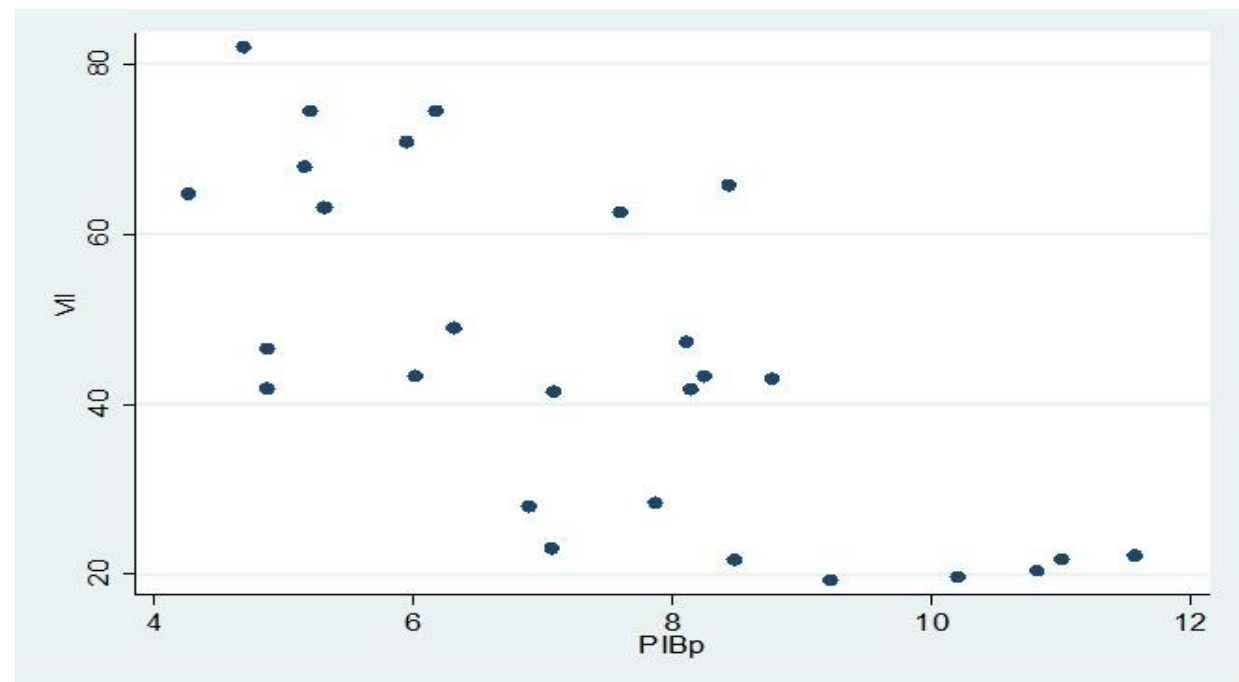

Figura 4 - Gráfico de Dispersão - MI - PIBp.

A mesma relação pode ser observada nas Figuras 5 e 6, onde um aumento em TFC reflete em um possível efeito de queda em MI, ou seja, poderia ocorrer melhorias dos aspectos socioeconômicos. Quando observado um aumento do IDH, vê-se que esse aumento poderia impactar em uma queda do número de mortalidade infantil. $\mathrm{O}$ que retoma no que foi dito anteriormente sobre as desigualdades sociais, quanto menores, menor será o número de mortalidade infantil. Essas relações são verificadas, em Ramalho (2014) [7] e Sousa (2016) [8], tanto o saneamento básico quanto os aspectos sociais e econômicos possuem relação com a mortalidade infantil, sendo as disparidades socioeconômicas sua principal causa. 


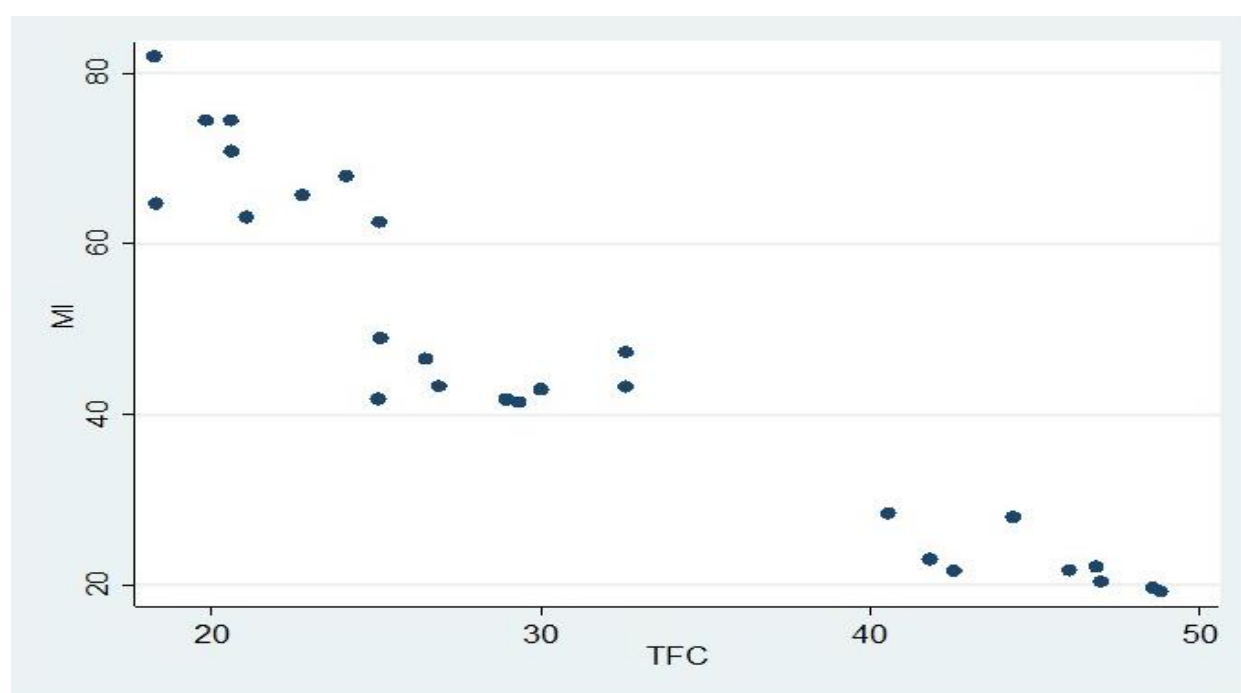

Figura 5 - Gráfico de Dispersão - MI - TFC.

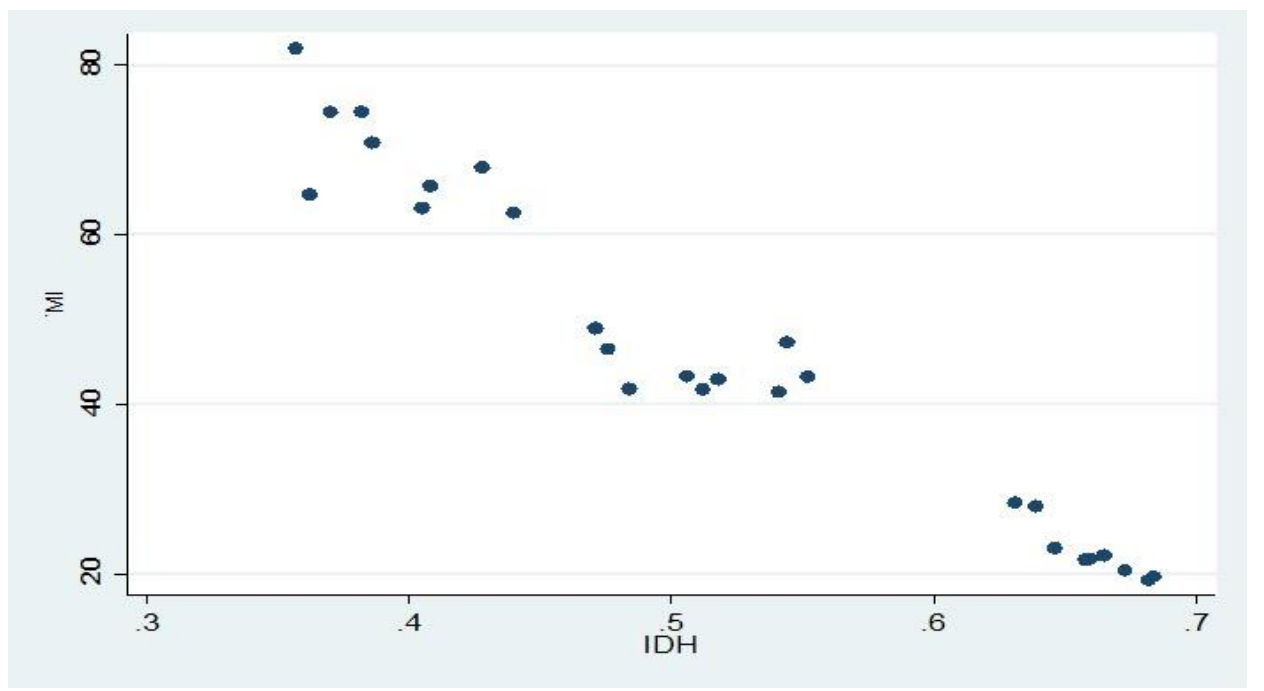

Figura 6 - Gráfico de Dispersão - MI - IDH.

\section{CONCLUSÕES}

Este estudo teve por objetivo analisar os impactos do saneamento básico sobre a mortalidade infantil e o desenvolvimento econômico da região Nordeste. Percebe-se por meio das análises realizadas, que os serviços de abastecimento de água e de esgotamento sanitário possuem relação direta com os números de mortalidade infantil, assim como as questões socioeconômicas. Vale ressaltar que a mortalidade infantil em si já é um indicador de qualidade de vida e de desenvolvimento.

Com base nas análises dos dados, foi possível observar que as regiões que possuem maiores índices de serviços inadequados de água e esgoto, são aquelas que também, possuem maior número de mortalidade infantil. No período em análise, houve diminuição dos serviços inadequados, o que refletiu em uma diminuição dos óbitos e das desigualdades sociais. Diminuição essa, que pôde ser observada pela melhoria do índice de desenvolvimento humano, ou seja, pela melhoria da educação, da renda e da saúde.

Pois, onde há melhoria da educação, ocorre maior procura pelos serviços de saneamento básico de qualidade, que se traduz na melhoria da saúde da população, tendo como reflexo a diminuição das doenças adquiridas pelo déficit dos serviços de saneamento básico e, também, a diminuição 
da mortalidade infantil, ocasionando melhorias nas condições socioeconômicas da população daquela localidade.

Destarte, pode-se concluir que o saneamento básico é um fator decisivo para a diminuição da mortalidade infantil, assim como a melhoria dos seus serviços impactam na melhoria do desenvolvimento econômico da região, pois uma população saudável é uma população mais produtiva.

\section{AGRADECIMENTOS}

A Universidade Federal de Sergipe (UFS).

\section{REFERÊNCIAS BIBLIOGRÁFICAS}

1. Leal FCT. Contexto e Prática da Engenharia Sanitária e Ambiental [Trabalho de conclusão de curso]. Universidade Federal de Juiz de Fora, Departamento de Engenharia Sanitária e Ambiental; 2012. 57 p.

2. Mendonça MJC, Motta RS. Saúde e Saneamento no Brasil. Planejamento e políticas públicas (IPEA). 2007 Jun/Dez;30:15-30.

3. Menezes TA, Uchoa F. Mortalidade Infantil, Saneamento Básico e o Impacto da Saúde sobre o Crescimento Econômico Brasileiro. 39 Encontro Nacional de Economia; 2011 Dez 6 - 9; Foz do Iguaçu (PR); v. 30, p.1-16.

4. Neri MC. Trata Brasil: saneamento e saúde. Rio de Janeiro: FGV, IBRE, CPS; 2007. 150 p.

5. Pasternak S. Habitação e saúde. Revista USP. 2016;30(86):51-66.

6. PNS. Política Nacional de Saneamento Básico [internet]. Lei $\mathrm{n}^{\circ} 11.445$ de 5 de janeiro de 2007. [Acesso em: 10 de outubro de 2016]. Disponível em: http://www.planalto.gov.br/ccivil_03/_ato20072010/2007/lei/111445.htm.

7. Ramalho WM. Desigualdades socioeconômicas e espaciais da mortalidade infantil no Brasil e Distrito Federal. [Tese]. Brasília (DF): Universidade de Brasília; 2014. 123 p.

8. Sousa JS. Estimação e Análise dos Fatores Determinantes da Redução da Taxa de Mortalidade Infantil no Brasil. Revista Brasileira de Estudos Regionais e Urbanos. 2016;10(2):140-155.

9. Turolla F. Novos Rumos para a Infraestrutura: Eficiência, Inovação e Desenvolvimento. $1^{a}$ edição. São Paulo: Lex Magister; 2014. Capítulo 6, Saneamento: O Despertar de um Setor Estagnado; p. 303-321. 